pass my hand into the pelvis, I found the vagina blocked up by a large mass, which was in fact the uterus completely inverted; having never seen anything approaching to a similar case, $I$ was at a loss how to act best for the safety of my patient. I had ordered the husband to call in one of my brother practitioners, and Mr. Jones was kindly in attendance in a very few minutes, whose account of the case I subjoin :-

"I saw Mrs. D. at forty minutes past eleven, P.M., found her pulseless, with her eyes turned up, and apparently sinking. I instantly placed her head in a depending position; upon applying $\mathrm{my}$ hand to the abdomen I missed the uterine tumour, and immediately suspected the uterus was inverted, which, upon examination, I found to be the case. My first impulse was to apply pressure to the most depending point, counter pressure being used with my right hand upon the abdomen, (where I could distinctly feel the os behind the pubes,) this produced no effect; I then grasped the uterus firmly for a short time, with the view of diminishing its size, and then applying the back of $m y$ fingers half closed to the upper and posteri.r part of the uterus, I was enabled gradually to return the part nearest the os, and when about half its volume was reduced, the fundus went up with a jerk."

A dose of ergot with brandy was now administered, the patient having previously swallowed half-a-pint of brandy in little more than a quarter of an hour; the pulse at the wrist became perceptible; the uterus contracted; a full dose of tincture of opium was given, and no untoward symptom has since occurred. I strictly probibited Mrs. D. from leaving her bed till after the third week. I have seen her this day, five weeks after her confinement, she could walk across the room without any symptom of prolapsus uteri, and quite as well as in the same period after her previous confinements.

I consider the alarming symptoms I before alluded to, after the expulsion of the placenta, to have been caused by the second shock the nervous system sustained from such an event as inversio uteri, and not from hamorrhage, which was by no means excessive.

\section{CASES IN PRIVATE PRACTICE.} By ROBERT MARTIN, Esa., Holbroox.

Read before the Suffolk Branch Meeting, held on the 25th of July, 1852.

Case of Calculary Deposits in the Excretory Ducts of the Sublingual Glands.

Ranula is by no means an uncommon disease of the sublingual glands, and must have fallen under the observation of many present, but the case I am about, (as briefly as possible) to lay before you, the complete obstruction of the excretory ducts on both sides the fronum linguæ by calculary deposit, is I believe someThat rare. I have never before seen an instance of it in my practice, which extends over a period of thirtyseven years.
Benjamin Page applied to me, stating that he had long suffered from "sore mouth," the fotor of which was intolerable, the whole mucous membrane of the mouth was highly inflamed, tongue swollen, and its point elevated by enlarged sublingual glands; at the bottom of a puffy swelling of the mucous covering of the right gland, was a small yellow spot, presenting the appearance of an ulcer, but on touching it with a probe, I was satisfied that it was a calculus. A free incision was made over it, and this calculus extracted. The left sublingual gland being even larger than the right, highly inflamed but not ulcerated, I made a free deep incision over it, the bistoury grated against a hard substance, which was for awhile, however, obscured by hæmorrhage, but on passing a probe into the wound, I readily picked out the calculus, measuring threefourths of an inch in length, and nearly an inch and a half in circumference. The wounds healed kindly, and in a few days the man was perfectly well.

\section{On Severe InJury of the KneE-Joint.}

The following case is one of practical interest, establishing the fact contended for by modern surgery, that severe injuries to large joints, with even extensive penetrating wounds into their cavities, do not invariably. require amputation.

John Rumsey, aged 37 years, while mowing grass; March 8th, 1849, fell with his bent knee on the edge of his scythe, which divided the whole anterior part of the joint, the ligamentum patellæ, with the external and internal lateral ligaments. To all appearance the leg hung by the integument on the back of the limb, the posterior ligaments, and flexor tendons. The patella was retracted, and a breadth of four fingers could be readily inserted between the head of the tibia and condyles of the femur; much blood was lost. Being from home, my partner, Mr. Jarmain attended, and the question of immediate amputation suggested itself, but being without assistance, he contented himself by placing the limb in as comfortable a position as possible till my return, with the heel well raised, and he brought the edges of the wound together with sutures, having previously freed them and the joint from coagula and all extraneous matters.

On visiting the case some hours after, If found him free from pain, the limb comfortable, and no constitutional disturbance present. All went on thus prosperously for three days, when heat and pain with profuse discharge, rendered it necessary to remove the dressings, the edges of the wound retracted, the sutures were sloughed out, the whole interior of the joint was exposed, and the fearful extent of the wound was fully apparent; extensive abscesses formed in the course of the vastus externus and biceps muscles, constitutional symptoms of disturbance set in-rigors, irritative fever, and profuse night sweats. These symptoms, however, gradually yielded to treatment, and after a confinement to his bed for fourteen weeks, to his house for twenty-six weeks, and an inability to return to his usual employment of 
eighteen months, he now again maintains a large family by his labour as a husbandman.

It will be noticed, that although great pains were taken during the progress of anchylosis to keep the leg straight, yet the inflammation and consequent shortening of the biceps, flexor, cruris, and outer head of the gastrocnemius, have produced a necessity in progression for eversion of the foot.

Although aware that Dr. Rhea Barton and Dr. Gibson, both of Philadelphia, are reported to have successfully operated for the removal of anchylosis of the knee-joint, I am too well satisfied with the result of this case to contemplate further interference.

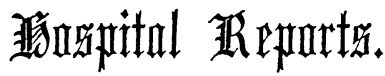

\section{WEST NORFOLK AND LYNN HOSPITAL.}

CASES ADMITTED UNDER THE CARE OF CHARLES COTTON, M.D. F.R.C.S., SENIOR SURGEON TO THE HOSPITAL.

\section{Stone in the Bladder-Lithotrity-Cure.}

WILLIAM KEMP, labourer, aged 64 years, admitted November 21st, 1848, with symptoms of stone of several month's duration. Catheter passed, and a moderate sized calculus detected.

Nov. 24th.-Bladder injected and screw lithotrite introduced. The stone was readily seized and easily crushed, the index of the instrument marking 9-12tbs, and yielding to 5-12ths on the application of the screw. On withdrawing the lithotrite the blades of the forceps were found densely impacted with triple phosphatic fragments. To keep his bed and have constant hot fomentations to pubic and perineal regions. Anodyne draught directly. Slop diet; barley, gum, or linseed mucilage for drink.

27th.-Several portions of detritus have come away during micturition, giving but little inconvenience. Catheter-forceps introduced, and some more fragments withdrawn. Anodyne draught given and fomentations repeated.

30th.-Muco-purulent and calculous deposit in the urine; "feels a little cutting on making water, and rather weak; but is otherwise quite well."-R. Bals. Copaibæ, m.xx. ; Liq. Potassæ, m.x.; Mucil. Acaciæ, oz., iss. Ter die sum. Full diet and gin.

December 5th-Made out-patient.

19th.-No difficulty attends micturition; urine clear; bladder carefully explored, and a catheter-scoop reintroduced, but not a particle of calculous matter discovered. Anodyne draught and hot fomentations. To remain an in-door patient a day or two. Repetatur mist. bis terve die.

30th.-Discharged cured.

\section{Stone in the Bladder-Eight small Calculi Removed by} Catheter-Forceps-Cure.

Samuel Fisher, farm labourer, aged 71 years. Admitted August 14th, 1849. Had suffered severely the last nine months, from pain referred to the region of the bladder, and great difficulty at times in making water, aggravated by walking, or riding in any uneasy vehicle. By sounding with the finger in the rectum, several small calculi were detected. The urinary passages were found in a highly sensitive condition, and the patient complained bitterly of the examination. To be immediately put to bed, to have flannels wrung out of hot water to the pubis, frequently renewed.-R. Extr. Hyoscyami, gr. ij.; Liq. Potassæ, m. xx.; Iufusi Diosmæ., oz. ij. M. Fiat haust stat. sum. et repetatur ter in die. Bread and milk or broth diet, and linseed decoction ad libitum.

18th.-Better, irritation subsided; bladder injected with warm water and catheter-forceps introduced. A small lithate of ammonia calculus the size of a horsebean, with its shell crushed, was then withdrawn between the blades of the instrument Scoop reintroduced, bladder injected through it and a second similar calculus extracted. Hot water applications to the perineum. No alteration in diet or medicine.

19th.-No unfavourable symptom; some soreness and smarting; has passed urine freely, mixed with a fer calculous particles and grit.

24th.-Three pisiform calculi removed by means of catheter-scoop.

September 1 st.-Three other small calculi, in all eight, extracted with the catheter-scoop.

2nd.-No material ailment beyond a trifling soreness. Urine thick and scanty. Full diet and porter.-R. Tinct. Ferri Sesquichlorid., m. x.; Tinct. Hyos., m. xx. ; Decoct. Pareiræ, oz. ij. Fiat haust. ter die sımendus.

7th.-Feels quite well and passes water without discomfort. Bladder explored; no other calculus detected. 10th.-Discharged.

Remarks. - The above cases (selected from upwards of a dozen occurring in hospital and private practice,) treated by the lithotrity or sliding catheter-forceps, without a single accident attending the operation, may be contrasted with that of Stebbing, reported in the Provincial Medical and Surgical Journal, of February, 1846 , a patient in whom the kidneys were found to be mere cysts, and where death took place on the 6th day, after a few "irresistible attempts" to break up large oxalate calculus.

The case of Fisher is destitute of any particular practical interest beyond that of showing the value of the sliding catheter-forceps, (invented by Mr. Weiss,) in cases where there is good reason to believe that the calculus is of small size, or when, after crushing, it is so desirable to prevent the chance of a fragment remaining to form a future nucleus. Indeed nothing can be more simple than the use of this instrument in cases to which it is especially applicable, if the following method be observed:-The injection of the bladder having been completed in the usual manner, and the stopcock-catheter withdrawn, the catheter-forceps, plugged, are to be introduced in its place, and the bladder fairly entered, the convexity of the fixed blade is then to be directed towards its posterior part, whilst the 